\title{
Combining tomographic imaging and DEM simulations to investigate the structure of experimental sphere packings $\dagger$
}

\author{
Gary W. Delaney, ${ }^{a b}$ T. Di Matteo ${ }^{b c}$ and Tomaso Aste ${ }^{* b c d}$ \\ Received 4th January 2010, Accepted 13th May 2010 \\ First published as an Advance Article on the web 3rd June 2010 \\ DOI: $10.1039 / b 927490 a$
}

\begin{abstract}
We introduce and describe in detail a "virtual laboratory" platform to study granular materials by combining advanced image reconstruction techniques from computed X-ray micro tomography and discrete element method simulations. This platform allows us to directly access quantities such as forces at the grain contacts, which would be otherwise hard to measure experimentally. We apply this technique to the investigation of equal sized bead packings prepared experimentally by means of different methods, and covering a broad range of packing fractions from $\phi=0.57$ to $\phi=0.64$. Results concerning the number of contacts, the distribution of forces at contact and the relation of these quantities with local and global packing properties are presented and discussed. This combined approach is found to both offer the ability to improve on previous tomographic measurements of geometric properties of the packings, and to estimate other physical properties that are not available experimentally.
\end{abstract}

\section{Introduction}

Everywhere we look in nature and the modern world we find granular matter. From sands to soils, grains to powders, our understanding of granular systems has applications in diverse areas of both fundamental and applied science ${ }^{1-3}$. In recent years, large efforts and several techniques have been developed and used to better understand the properties of these materials. In particular one of the most important experimental innovations in this field has been the introduction of imaging techniques such as X-ray computed tomography (XCT) ${ }^{4}$ nuclear magnetic resonance (NMR), ${ }^{5}$ confocal microscopy, ${ }^{6}$ refractive index matching ${ }^{7}$ and photoelastic methods, ${ }^{8}$ which allow scientists to directly visualize the internal structure of granular assemblies and measure some of their relevant properties. In conjunction with this progress in experimental techniques, large scale computational studies have also become more tractable, with the ready availability of increasing computational power and sophisticated simulation tools. In this paper we describe the development of a virtual laboratory platform that combines direct imaging data from $\mathrm{XCT}^{9,10}$ with a computational model based on the discrete element method (DEM). ${ }^{11,12}$

Previous computational studies of granular systems have incorporated several simulation techniques, including Monte Carlo type models, ${ }^{13}$ lattice models,${ }^{14}$ energy minimisation

${ }^{a}$ CSIRO Mathematical and Information Sciences, Private Bag 33, Clayton South, Vic, 3168, Australia

${ }^{b}$ Department of Applied Mathematics, School of Physical Sciences, The Australian National University, Canberra, ACT 0200, Australia

'Department of Mathematics, King's College London, The Strand, London, $W C 2 R 2 L S, U K$

${ }^{d}$ School of Physical Sciences, University of Kent, Canterbury, Kent, CT2 7NH,UK.E-mail: t.aste@kent.ac.uk

$\uparrow$ This paper is part of a Soft Matter themed issue on Granular and jammed materials. Guest editors: Andrea Liu and Sidney Nagel. methods,${ }^{15}$ and the discrete element method. ${ }^{16,17}$ From a computational perspective, among these many models which have been proposed and used in the literature, ${ }^{18}$ the most versatile, realistic and nowadays common approach in use is DEM ${ }^{1,2,19-22}$ and good agreement has been found between predictions of DEM simulations and experiments. ${ }^{23}$

From the experimental side, XCT is an impressive and versatile tool that provides a digital map of the internal structure of real granular systems, with a resolution that can reach $1 \mu \mathrm{m}$. However, some information such as the inter-grain forces cannot be directly retrieved from a pure geometrical characterization of the system structure. In this respect, DEM offers the ability to better characterize the system under consideration, allowing access to all its properties down to the forces on individual grains and at inter-grain contacts. However, this is at the expense of some simplifications of the physics that depend on the exact details of the chosen DEM model and also the general limitation of idealized grain geometries (perfectly spherical in this case).

In our approach, we use a tomographic reconstruction of a real sphere packing as our starting point (see Fig. 1), and input this experimental data (in the form of the coordinates of the grain centers) into our DEM simulation. ${ }^{11,12}$ Specifically, we apply this technique to a set of experiments ${ }^{24}$ concerning equalsized beads in air and in water prepared using different materials and methods. ${ }^{9,11,12,25-27}$ In a typical simulation, the spheres re-arrange from the initial positions by moving only a small fraction of their diameters to settle into the final configuration where force and torque are balanced and the system rests in mechanical equilibrium. By means of this numerical relaxation we remove the experimental uncertainty about the exact location of the sphere centers associated with the limited XCT resolution and also we remove the uncertainty about the exact sizes of the experimental beads associated with variations in the grain sizes (polydispersity) and deviations from sphericity. From the experimental bead pack, we thus produce a simulated 
ideal mono-disperse sphere packing, a 'virtual packing', that matches the original geometry of the experimental bead pack and that can now be used to compute with precision several static and dynamical properties which are otherwise not directly accessible from experiments. In this paper we describe this virtual platform and demonstrate its application by investigating several properties of granular packings, including: the number of grains in contact, the distribution of forces at contact and the relation between these properties and the local and global packing fractions.

The paper is organized as follows. In section II, we briefly describe the experimental methods and materials. In section III, the main equations and methods implemented in the DEM simulations are reviewed. In section IV, the parameters used in the dynamical relaxation of the experimental samples are provided and the relaxation dynamics are described. In section $\mathrm{V}$, we discuss changes in the radial distribution function between the original experimental samples and the DEM relaxed data. In section VI, the number of contacts in the DEM relaxed systems are studied in detail and a comparison with previous estimations by means of a different method is discussed. In section VII, we investigate the relation between the number of contacts and the packing fraction both locally and globally, discussing the validity of theoretical predictions. In section VIII, the isostatic condition is discussed and the minimal size of clusters satisfying the isostatic condition is investigated. In section IX, we report the distribution of the number of contact per sphere and we provide a simple statistical mechanics derivation of these distributions. In section $\mathrm{X}$, we investigate the distribution of normal forces at contact and discuss the relation with local packing properties. Finally in section XI, conclusions and perspectives are provided.

\section{Experiments}

We use the AAS database on disordered packings ${ }^{24}$ which contains structural data from experimental sphere packings obtained by X-ray computed tomography.

\section{A. Dry acrylic beads in air}

Specifically, we analyze 6 samples (A-F) composed of dry acrylic beads in air poured (by hand) into a cylindrical polycarbonate container with an inner diameter of $55 \mathrm{~mm}$ and filled to a height of $\sim 75 \mathrm{~mm} . .^{9,25,28}$ To reduce boundary effects, the inside of the cylinder was roughened by randomly gluing spheres to the internal surface. Samples A and C contain $\sim 150000$ beads with diameters $d=1.00 \mathrm{~mm}$ and polydispersity within $0.05 \mathrm{~mm}$. Samples B, D-F contain $\sim 35000$ beads with diameters $d=1.59$ $\mathrm{mm}$ and polydispersity within $0.05 \mathrm{~mm}$. The two samples at lower packing fraction (A, B) were obtained by placing a stick in the middle of the container before pouring the beads and then slowly removing the stick. ${ }^{21}$ Sample C was prepared by gently and slowly pouring the spheres into the container, while sample D was obtained by a faster pouring. In sample E, a higher packing fraction was achieved by gently tapping the container walls. The densest sample (F) was obtained by a combined action of gentle tapping and compression from above (with the upper surface left unconfined at the end of the preparation). The geometrical investigation of the packing structure was performed over a central region at 4 sphere-diameters away from the sample boundaries. These were the first samples analyzed ${ }^{28}$ and this kind of preparation "by hand" was devised to mimic the real-world handling of granular materials, since as discussed in ref. 25 , we were interested in properties that are little affected by the details of the preparation. However, we have been aware that this kind of preparation method might produce misleading non-reproducible results. For this reason we devised a second set of experiments where, on the contrary, the experimental handling is highly controlled and the outcomes readily reproducible.

\section{B. Glass beads in water}

This new set of experiments uses a fluidized bed technique. ${ }^{27,29}$ This technique is typically comprised of a vertical column containing a fluid and a bed of granular material supported on a porous plate through which the fluid can flow upwards. During a flow pulse the grains expand and the particles move upward randomly. After the pulse ends, the bed sediments down into a new mechanically stable configuration. Packings created in this way are in a stationary state, with packing fractions that have average values which depend on the flow rate, with lower packing fractions obtained for higher flow rates (larger bed expansions). In this paper we analyze 12 samples (FB14-24 and FB27) prepared by means of this technique. These samples contain about 150000 glass beads with diameters $0.25 \mathrm{~mm}$ placed in a vertical polycarbonate tube with an inner diameter of $12.8 \mathrm{~mm}$ and a length of $230 \mathrm{~mm}$. The packings were prepared in water. ${ }^{24,26,29}$ Packing fractions between 0.56 and 0.60 were obtained using different flow rates.

\section{X-Ray computed tomography imaging}

We use X-ray computed tomography $(\mathrm{XCT})^{4}$ to calculate the coordinates of the bead centers. The outcome of an XCT scan is a three dimensional density map, ${ }^{67}$ discretised in space as a set of voxels (3-dimensional volume element analogous to the 2dimensional pixel). Providing the granular material has
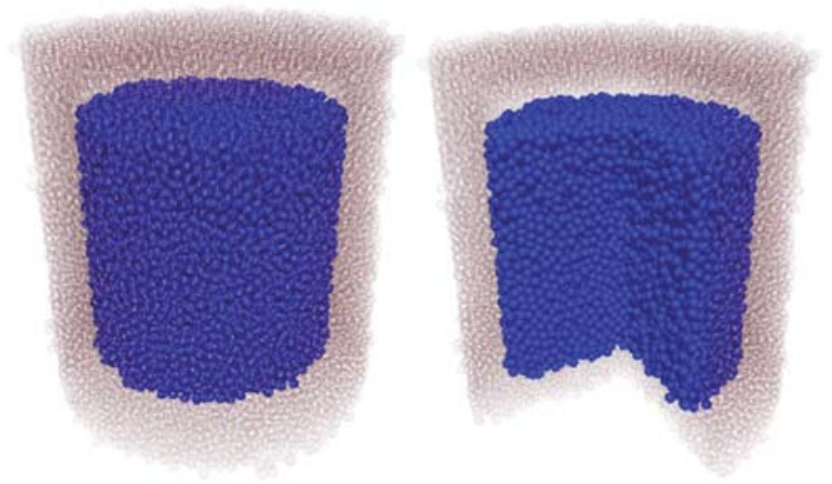

Fig. 1 Combined tomographic and DEM reconstruction of a sphere packing. The inner spheres that are part of the DEM simulation are rendered opaque whereas the fixed boundary spheres are rendered translucent. Images are for the F sample with 6856 spheres in the internal region. The entire sample is shown (left) and also the sample with one quarter removed (right) to better show the internal structure of the packing. 
a different X-ray attenuation than the surrounding media, then the map reveals the discretized 3-dimensional shape of the grains. In the facility at the Australian National University that we used for these experiments, ${ }^{30,31}$ the highest resolution is around $1 \mu \mathrm{m}$, with the 3 -dimensional map consisting of $2024^{3}$ voxels. Our XCT apparatus uses a cone beam geometry ${ }^{30,31}$ and the actual resolution in a given experiment depends on the relative position of the sample with respect to the X-ray source. Specifically the voxel side size in our sets of experiments are: $0.0399 \mathrm{~mm}$ (samples A, C), $0.0516 \mathrm{~mm}$ (samples B, D, F) and $0.0172 \mathrm{~mm}$ (for all the FB14-24, FB27 samples). The use of spherical grains of equal sizes highly simplifies the image processing because it is necessary to retrieve only the information concerning the grain center. This is done by applying a convolution method over the threedimensional XCT density map and this is made algorithmically very efficient by using (parallel) fast Fourier transform. Furthermore, in combination we used the watershed method ${ }^{32}$ (which is a segmentation algorithm based on the image topology) to identify distinct grains. The resulting bead positions are estimated with a sub-voxel precision better than $0.1 \%$ of the beads diameters. Indeed, the position of the center of mass can be estimated with higher precision than the size of the voxels in the density map because these coordinates are calculated as a center of mass of the digitalized bead volume that contains several hundreds voxels. This precision is well below the beads' polydispersity which is between $1 \%$ and $3 \%$ considering both changes in the bead sizes and deviations from perfect sphericity. ${ }^{68}$

\section{Simulation}

The discrete element method (DEM) has been employed extensively in simulations of granular systems $s^{1,2,11,12,19-21}$ and here we employ it to simulate a system of frictional, viscoelastic grains under the influence of gravity $\left(g=9.81 \mathrm{~ms}^{-2}\right)$. Grains interact only in compression, with a normal repulsive force $F_{n}$ accordingly with Hertz's theory ${ }^{33}$

$$
F_{n}= \begin{cases}k_{n} \xi_{n}^{3 / 2} & \text { for } \xi_{n} \geq 0, \\ 0 & \text { for } \xi_{n}<0\end{cases}
$$
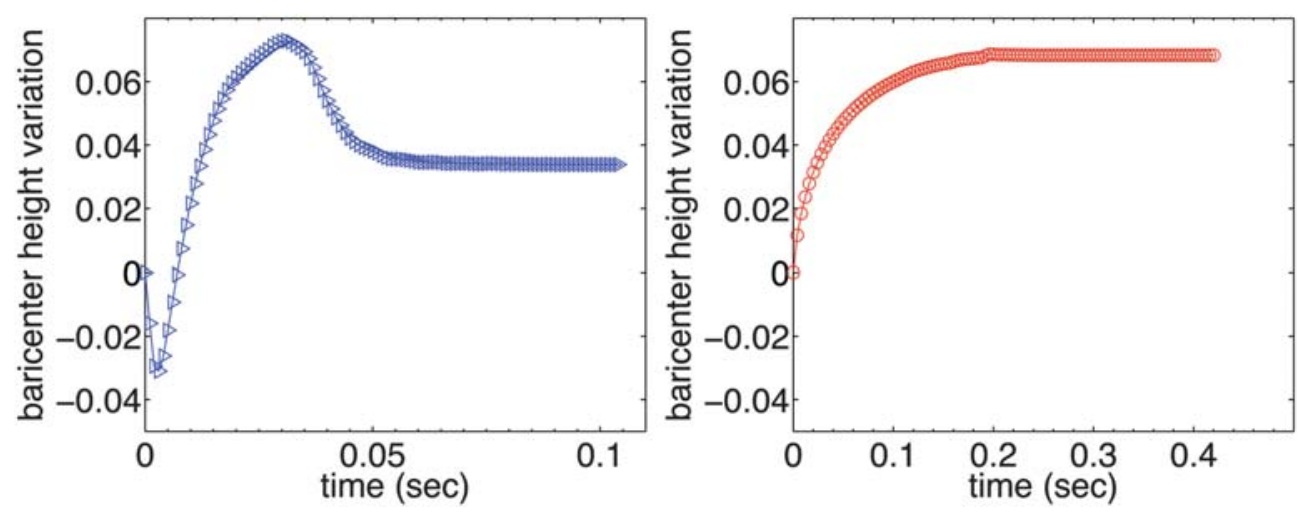

Fig. 2 Variation of the barycenter height (in units of $d$ ) during a DEM relaxation simulation. The left plot refers to the sample with lowest packing fraction in the fluidized bed experiments (sample FB18) and the right plot is the sample with the highest packing fraction in the dry beads experiment (sample F). with $\xi_{n}=d-\left|r_{i}-r_{j}\right|$, where $d$ is the sphere-diameter and $r_{i}$ and $r_{j}$ are the positions of the grain centers. ${ }^{20,34,35}$ The spring constant $k_{n}$ is related to the Young's modulus $E$ and Poisson's ratio $\nu$ of the grains, with $k_{n}=\frac{d^{1 / 2} E}{3\left(1-\nu^{2}\right)}$. We also consider the tangential force $F_{t}$ under oblique loading with

$$
F_{t}=-k_{t} \xi_{n}^{1 / 2} \xi_{t}
$$

where $\xi_{t}$ is the elastic tangential displacement between spheres, obtained by appropriately integrating the tangential relative velocities during the lifetime of the contact, subject to the constraint that $\xi_{t}$ is truncated such that $F_{t}$ cannot exceed the Coulomb friction criteria $\left|F_{t}\right| \leq \mu\left|F_{n}\right|{ }^{19,36,39}$ For the simulations presented here we take the commonly used approach of setting $k_{t}=\frac{2}{7} k_{n}$ such that the normal and tangential characteristic collision times are equal. ${ }^{37,38} \mathrm{We}$ also include a normal viscoelastic dissipation

$$
F_{n}=-\gamma_{n} \xi_{n}^{1 / 2} \dot{\xi}_{n}
$$

where $\dot{\xi}_{n}$ is the normal velocity. A viscous friction force is also included in the model $^{38}$

$$
F_{t}=-\gamma_{t} v_{t}
$$

where $v_{t}$ is the tangential component of the relative grain velocity. The values used for $k_{n}, k_{t}, \gamma_{n}$ and $\gamma_{t}$ are discussed in the next section.u

\section{DEM relaxation of experimental samples}

In order to eliminate the unavoidable experimental incertitude in the bead positions, sizes and shapes, we reconstruct a numerical sample of equal spheres. This is done by performing a DEM relaxation simulation, initialised from the experimental bead coordinates measured by XCT imaging.

The DEM simulation is performed on a set of unfixed spheres in the central region of the sample, with the boundaries provided by the outer spheres which are kept fixed at the positions measured via XCT experiment. Three layers of spheres are 
removed from the top of the unfixed sphere set allowing the spheres to rise freely in height if necessary. We use realistic physical parameters for acrylic beads having: Young modulus $3.2 \mathrm{GPa}$; Poisson ratio 0.3 ; density $1150 \mathrm{~kg} / \mathrm{m}^{3}$; and inter grain static friction coefficient 0.28 . Samples $\mathrm{A}, \mathrm{C}$ have radius $0.5 \mathrm{~mm}$ whereas samples B,D,E,F have radius $0.795 \mathrm{~mm}$. For the glass beads we used: Young modulus $70 \mathrm{GPa}$, Poisson ratio 0.2; density $2500 \mathrm{~kg} / \mathrm{m}^{3}$; inter grain wet static friction coefficient 0.9 and radius $0.125 \mathrm{~mm}$. All the simulations use a simulation time step equal to $\Delta t=10^{-7} \mathrm{~s}$.

To ensure a purely quasi-static relaxation, large values of the normal and tangential dissipation parameters $\gamma_{n}$ and $\gamma_{t}$ were used. Values in the range from $\gamma_{n}=\gamma_{t}=10$ to 100 were considered, with the properties of the relaxed structure being found to be insensitive to the exact choice of value. We also verified that during the relaxation process grains keep an average number of contacts larger than 4. Essentially, the relaxation dynamics consists in a re-equilibration of the elastic stress trapped in the grain overlaps in the initial configurations which are unphysical and unbalanced and are due to the experimental uncertainties on the grain positions, grain polydispersity and asphericity. The viscoelastic dissipation and viscous friction remove energy from the system until the spheres have all settled down to a stationary state. We consider that the system has achieved a stationary condition when kinetic energy is below $10^{-10}$ times the maximum value (reached shortly after the beginning of the relaxation) and when overlap energy is unchanged up to the 15 th digit.

Fig. 2 shows the variation in the average height of the grains as the simulation proceeds. Typically during the DEM relaxation
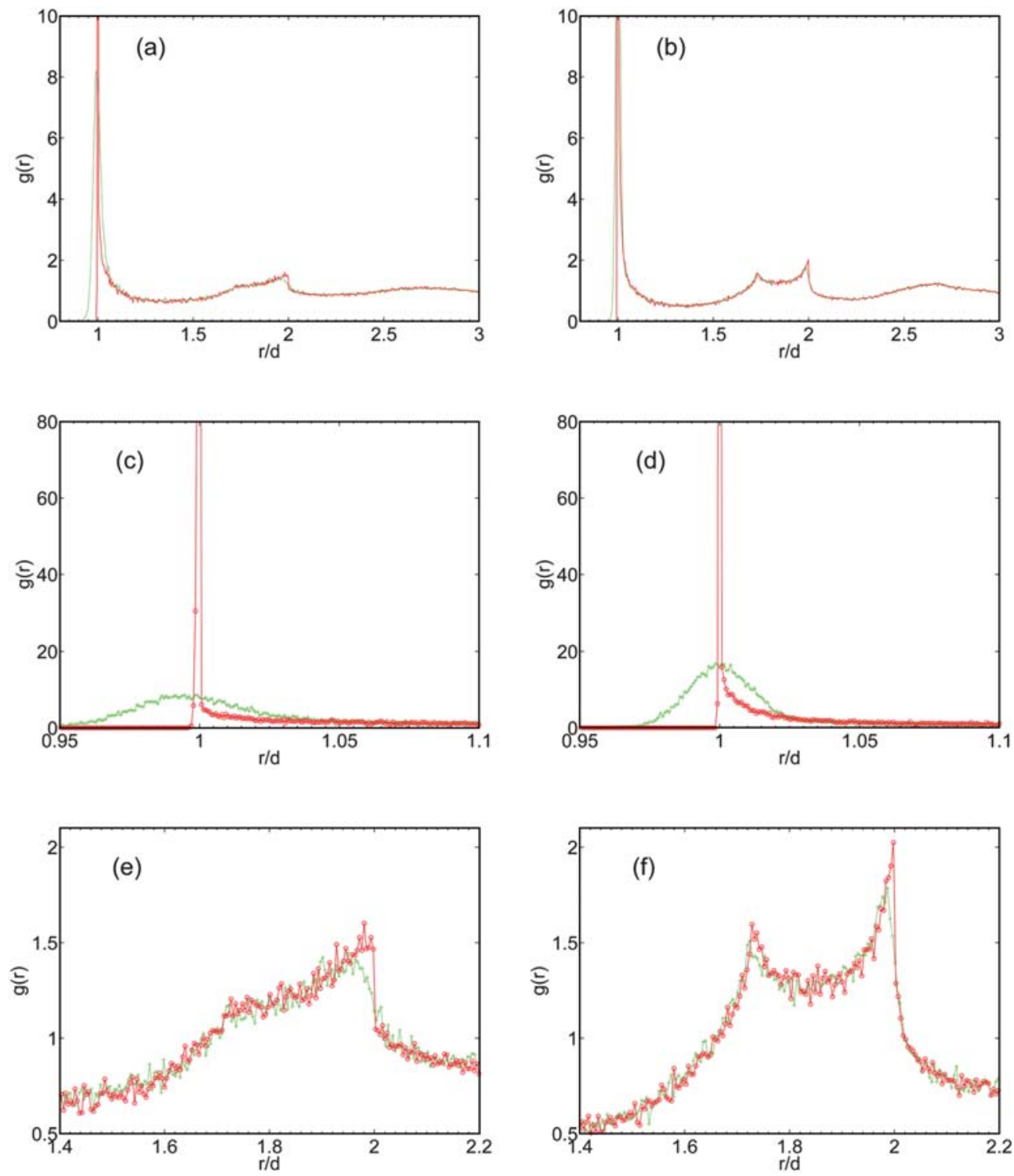

Fig. 3 The data refer respectively to (left plots: a,c,e) the sample with lowest packing fraction in the fluidized bed experiments (sample FB18) and (right plots: $b, d, f)$ the sample with the largest packing fraction in the dry beads experiment (sample F). Figures (c,d) show the detail of the first peak. Figures $(\mathrm{e}, \mathrm{f})$ show the detail of the second and third peaks. The '+' symbols refer to XCT data and the ' $O$ ' symbol to DEM relaxed systems. It is clear that all peaks become sharper in the DEM relaxed systems. 
there is initially a small expansion of the system, with the height increasing by a fraction of percent of the initial height. The final average height of the grains is within $0.1 \%-0.2 \%$ of the initial average height (the average height is taken measuring the distance, in units of $d$ between the lowest mobile sphere and a top layer of the 30 highest mobile spheres). The average displacement of the centers of the spheres during the relaxation process is less than $5 \%$ of the sphere diameters.

It is important to stress that in the simulations the stationary state is reached in less that a second (simulation time) with the spheres moving only a small fraction of their diameters. Such a short simulation time allows us to relax these rather large systems using realistic stiffness values.

\section{Radial distribution function}

A classical quantity widely used to characterize the structural organization of packings is the radial distribution function $g(r)$ which is associated to the probability of finding the center of a sphere in a given position at a distance $r$ from a reference sphere. This quantity is calculated by counting the number of sphere centers within a radial distance $r$ from a given sphere center ( $z(r)$, see Fig. 4) and using the equation ${ }^{69}$

$$
z\left(r_{1}\right)-z\left(r_{0}\right)=\int_{r_{0}}^{r_{1}} g(r) 4 \pi r^{2} d r .
$$

Although there are more specific and better devised methods to investigate and characterize the geometrical organization in disordered packings, ${ }^{25}$ the comparison between $g(r)$ from the original and the DEM relaxed samples is a good way to measure average variation in the internal structure. Fig. 3 shows the radial distribution function $\tilde{g}(r)$ (which has been normalised such that $\tilde{g}(r) \rightarrow 1$ for $r \rightarrow \infty$ ) for both the DEM relaxed samples and the original tomographically obtained data. We observe that both sets of data show a peak at $r=d$, which is however much sharper in the DEM simulation. We also observe two peaks at $r=\sqrt{3} d$ and $r \simeq 2 d$, which are again sharper in the DEM simulation but they preserve the overall characteristics of the experimental data. This is a strong indication that there are essentially no differences between geometrical properties in the original experimental samples and in the DEM relaxed samples except for the acquired
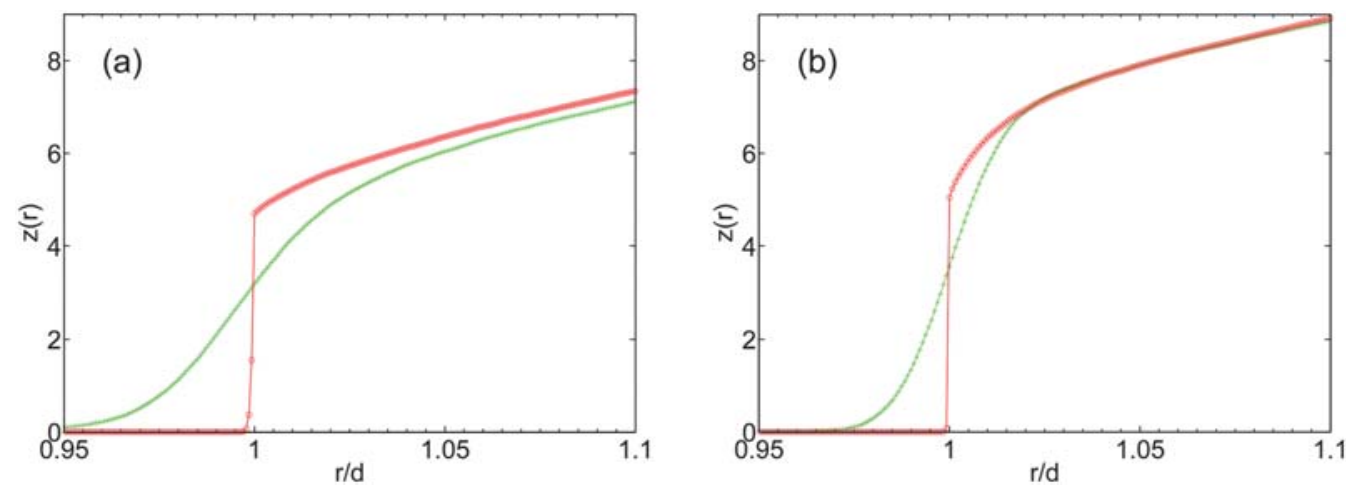

Fig. 4 Average number of sphere centers within a distance $r$ from the centre of a sphere. The DEM relaxed samples (circles) reveal a sharp discontinuity at $r=d$ whereas the original XCT data (squares) reveal a smoother transition. The left plot refers to the sample with lowest packing fraction in the fluidized bed experiments (sample FB18) and right to the sample with the largest packing fraction in the dry beads experiment (sample F). The fact that $z(r)$ for the XCT experimental data does not vanish until a few per-cent below $r=d$ is consistent with the grain polydispersity which is indeed estimated about $5 \%$ for the acrylic beads and $3 \%$ for the glass beads.
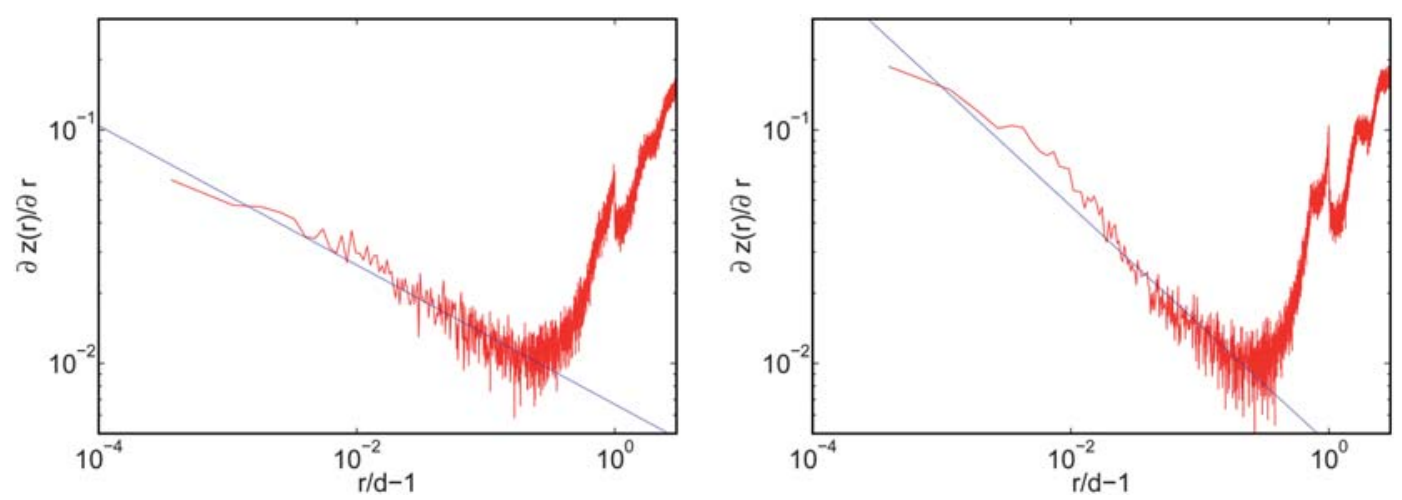

Fig. 5 The rate of variation of the number of neighbours $v s$. radial distance shows power law behaviors: $\partial z(r) / \partial r=(r-d)^{\alpha-1}(\operatorname{linear} \operatorname{trend}$ in $\log -\log$ scale in the region $r \in[d, 1.4 d]$ ). (Left) Fluidized bed sample FB18; (right) dry acrylic sample F. The exponent depends on sample preparation and packing fraction (see Fig. 6). The straight lines are the best fits in the region $r \in[d, 1.4 d]$. 
uniformity of the sphere diameters. We must stress that the appearance of these peaks does not necessarily indicate any presence of translational order, they are simply associated with repetition of structural motifs. This point is discussed in ref. 25.

\section{A. Power law increase in the number of neighbors}

The average number of neighbors $z(r)$ that are within a given radial distance $r$ from a sphere centre in the packing is a quantity directly related to $g(r)$ through eqn (5). For a packing of perfect non-overlapping spheres, $z(r)$ must be identically equal to zero for any $r<d$ and then it must jump to the number of neighbors in contact $z_{c}$ at $r=d$. In Fig. 4 we can see that $z(r)$ from the experimental samples has a smooth increase in value beginning at distances up to $5 \%$ below $r=d$. A very different behavior is revealed by the DEM relaxed samples where a jump to $z_{c}$ is clearly visible at $r=d$.

For larger distances, $z(r)$ keeps growing smoothly as other centers from nearby spheres that are not in contact become counted. It has been pointed out in the literature ${ }^{40,41}$ that, in a region after the jump at $r=d$, the number of neighbors within a given radial distance $z(r)$ increases with the distance $r$ accordingly with the law (for $r \in[d, 1.4 d]$ ):

$$
z(r)=z_{c}+z_{1}(r-d)^{\alpha}
$$

which implies

$$
\frac{\partial z(r)}{\partial r}=z_{1} \alpha(r-d)^{\alpha-1}
$$

It has been argued that such a power law behavior has interesting relations with the statistical physics of these systems and it is related to the dynamical arrest at the jamming transition. ${ }^{41-43}$

Fig. 5 reports $\partial z(r) / \partial r v s$. $r$ for the two samples with respectively the smallest and largest packing fractions (FB18 and F). As one can see both the samples show a good linear trend in the loglog scale revealing that eqn (7) is followed. Similar behaviors are observed for all samples. However, we find that the exponents $\alpha$ are not universal. Indeed, they change with packing fraction and depend on the sample preparation as shown in Fig. 6. This is in apparent contradiction with the results and discussions in ref.

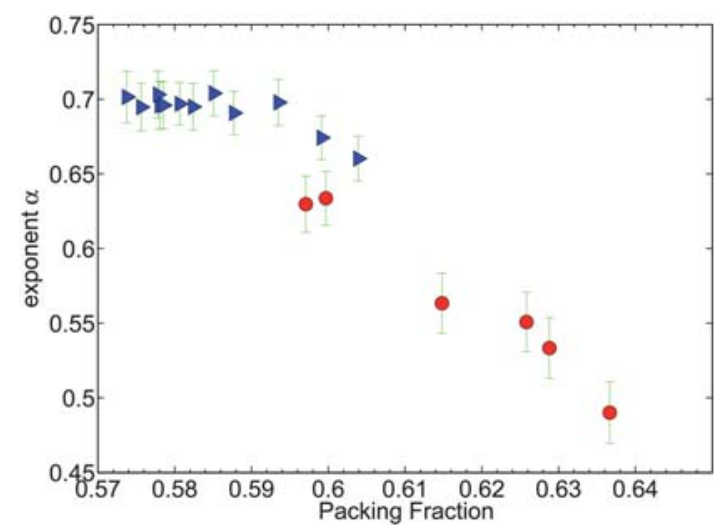

Fig. 6 Exponent $\alpha v$ s. packing fraction for all the samples. The error bars represent $95 \%$ confidence interval over the estimation of $\alpha$ from linear regression. Here the triangles ' $\triangle$ ' refer to all the fluidized bed samples and the circles ' $O$ ' to the dry acrylic bead samples.
$15,40,41$ where lower values of the exponents are found and a 'universal' value of $\alpha=0.5$ is suggested. However, it must be stressed that numerical results in ref. 40 uses hard sphere potentials and ref. 15,41 refer to non-frictional spheres. These are systems that can jam only at packing fraction 0.64 . On the contrary, our system is frictional and therefore it is different from the system studied in ${ }^{15,40,41}$, furthermore, from Fig.6 one can see that our data are consistent with $\alpha=0.5$ at $\Phi=0.64$.

\section{Number of contacts}

The average number of neighbors in contact with any given sphere in the packing $\left(z_{c}\right)$ is a simple and important measure of the system's topological structure which has been used since the first investigation of these systems. ${ }^{21,45-55}$ This quantity has recently become central in the theoretical description of granular systems by means of statistical mechanics arguments. ${ }^{44}$ The estimation of this quantity from geometrical structural measurements is extremely problematic because grains can be infinitesimally closed but not in mechanical contact. This problem can only be solved if one can measure the forces between grains. Direct observation of the stress with photoelastic materials is possible but it is extremely challenging in three dimensions and, to our knowledge, there is still no available data. On the other hand, with tomographic data we cannot measure directly the forces between grains, however by DEM relaxing the systems we can. Indeed, one of the main initial motivations for the present study was to provide a precise measurement of the number of grains in contact.

Fig. 4 shows the comparison between $z(r)$ from the DEM relaxed samples and the original tomographic data. It is clear that the determination of the average number of neighbors in contact (often referred in the literature as 'coordination number') from the original experimental data is an extremely difficult task because the data has a slow smooth increase in the number of neighbours over a range of $r=0.97 d \rightarrow 1.02 d$. Conversely, the DEM relaxed samples show a much sharper increase in the number of neighbors, with a discontinuity at $r \simeq d$ giving a good estimate of the actual

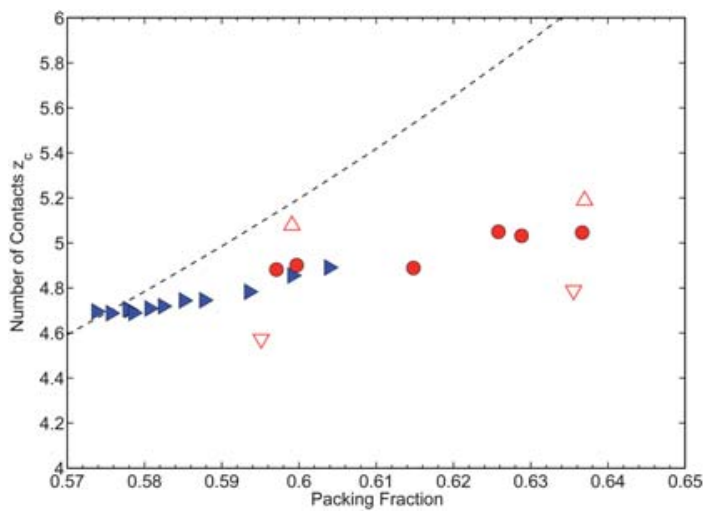

Fig. 7 Average number of neighbors in contact $v s$. packing fraction for all the experimental samples: fluidized bed $(\triangleright)$ and dry acrylic beads $(\bigcirc)$. The dashed line is the theoretical prediction from ref. 44 $z_{c}=2 \sqrt{3} \Phi /(1-\Phi)$. The symbols $\nabla$ refer to two samples (B and F) relaxed with a larger friction coefficient $(\mu=0.9$ instead of 0.28$)$. Conversely, the symbols $\Delta$ refer to samples B and $F$ relaxed with a smaller friction coefficient $(\mu=0.2)$. 
Table 1 Details for the internal region of the DEM relaxed fluidized bed samples: number of internal mobile spheres $(N)$, packing fraction $(\Phi)$, average number of neighbors $\left(z_{c}\right)$, fraction of contacts that cannot hold extra tangential force $(q)$, prediction for the isostatic condition from eqn $(15)$ ( $\left.z_{I S O}\right)$, critical size $\left(l^{*}\right)$ in units of $d$

\begin{tabular}{|c|c|c|c|c|c|c|c|c|c|c|c|c|c|}
\hline Sample & $N$ & $\Phi$ & $z_{c}$ & $q$ & $z_{I S O}$ & $l^{*}$ & Sample & $N$ & $\Phi$ & $z_{c}$ & $q$ & $z_{I S O}$ & $l^{*}$ \\
\hline FB18 & 6857 & 0.5738 & 4.70 & 0.026 & 4.04 & 11.8 & B & 7041 & 0.5971 & 4.88 & 0.150 & 4.21 & 11.6 \\
\hline FB17 & 7242 & 0.5756 & 4.69 & 0.027 & 4.04 & 10.7 & A & 6922 & 0.5997 & 4.90 & 0.163 & 4.23 & 11.0 \\
\hline FB15 & 7041 & 0.5778 & 4.71 & 0.025 & 4.03 & 10.3 & $\mathrm{C}$ & 6804 & 0.6148 & 4.89 & 0.143 & 4.20 & 9.8 \\
\hline FB14 & 6922 & 0.5780 & 4.70 & 0.027 & 4.04 & 9.2 & $\mathrm{D}$ & 7242 & 0.6258 & 5.05 & 0.162 & 4.23 & 8.7 \\
\hline FB20 & 6746 & 0.5785 & 4.69 & 0.022 & 4.03 & 9.4 & $\mathrm{E}$ & 6857 & 0.6288 & 5.03 & 0.152 & 4.21 & 9.4 \\
\hline FB16 & 6804 & 0.5806 & 4.71 & 0.029 & 4.04 & 10.4 & $\mathrm{~F}$ & 6856 & 0.6367 & 5.05 & 0.169 & 4.24 & 9.3 \\
\hline FB19 & 6856 & 0.5824 & 4.72 & 0.030 & 4.04 & 11.6 & & & & & & & \\
\hline FB21 & 6927 & 0.5851 & 4.74 & 0.027 & 4.04 & 10.7 & & & & & & & \\
\hline FB22 & 6944 & 0.5877 & 4.75 & 0.028 & 4.04 & 10.3 & & & & & & & \\
\hline FB23 & 6890 & 0.5935 & 4.78 & 0.028 & 4.04 & 10.3 & & & & & & & \\
\hline FB27 & 7214 & 0.5991 & 4.86 & 0.030 & 4.04 & 10 & & & & & & & \\
\hline FB24 & 7054 & 0.6039 & 4.89 & 0.031 & 4.04 & 9.3 & & & & & & & \\
\hline
\end{tabular}

average number of contacts $z_{c}$. Such an estimate of the number of contacts for all the DEM relaxed samples is shown in Fig. 7. We observe a linearly increasing trend in the number of contacts with the packing fraction with the two extremes being $z_{c} \sim 4.7$ at $\Phi \sim$ 0.57 to $z_{c} \sim 5.0$ at $\Phi \sim 0.64$. We also observe little dependence on the preparation method with the fluidized beds and dry bead samples having comparable values at the same packing fractions. Table 1 reports the values of $z_{c}$ and $\Phi$.

\section{A. Number of near neighbors}

Beside the spheres in contact, there are a large number of other spheres that are near but do not touch. These 'near neighbors' certainly play a significant role in the formation of the actual packing configuration and provide the necessary environment to guarantee mechanical stability upon small perturbations. There is however some arbitrariness in counting such neighbors. Indeed, as clearly visible from Fig. 4, there is a steep increase in the number of sphere centers with radial distances immediately above $r=d$.

An interesting perspective on the analysis of the number and the role of near neighbors has been recently proposed in ref. 44 by using a modified radial distribution function which counts the average value of the number of grains in contact with a spherical surface radius $r$ renormalized by the factor $(d / 2)^{2} / r^{2}$ (which is the ratio between surface area of a bead and the surface area of a sphere of radius $r$ ). In our notation such a quantity is (for $r \geq d / 2$ ):

$$
g_{z}(r)=\frac{d^{2}}{4 r^{2}}[z(r+d / 2)-z(r-d / 2)]
$$

At $r=d / 2$ this quantity coincides with $z_{c}=z(d)$, but then it has the peculiar property of showing a maximum at a radial distance which is very near (within a fraction of percent) to the contact point. Such a behavior is shown in Fig. 8. From a mathematical perspective such a maximum is the consequence of the power law growth of $z(r)$ (see eqn (6)) which combined with the factor $1 / r^{2}$ gives first a growing trend near $r=d / 2$ and then a decreasing behavior at larger $r$. The location of such a maximum depends on the parameters $z_{c}, z_{1}$ and $\alpha$, but the typical position is within $0.5 \%$ from $r=d / 2$. Indeed, from Fig. 8a, it is clear that in our samples the position of the maximum of $g_{z}(r)$ varies in a relatively narrow range between $r-d / 2=0.0035$ and $r-d / 2=0.0065$.

It has been argued in ref. 44 that a good estimation of the neighbors in near contact is given by $g_{z}(r)$ at $r-d / 2=0.004 d$. In
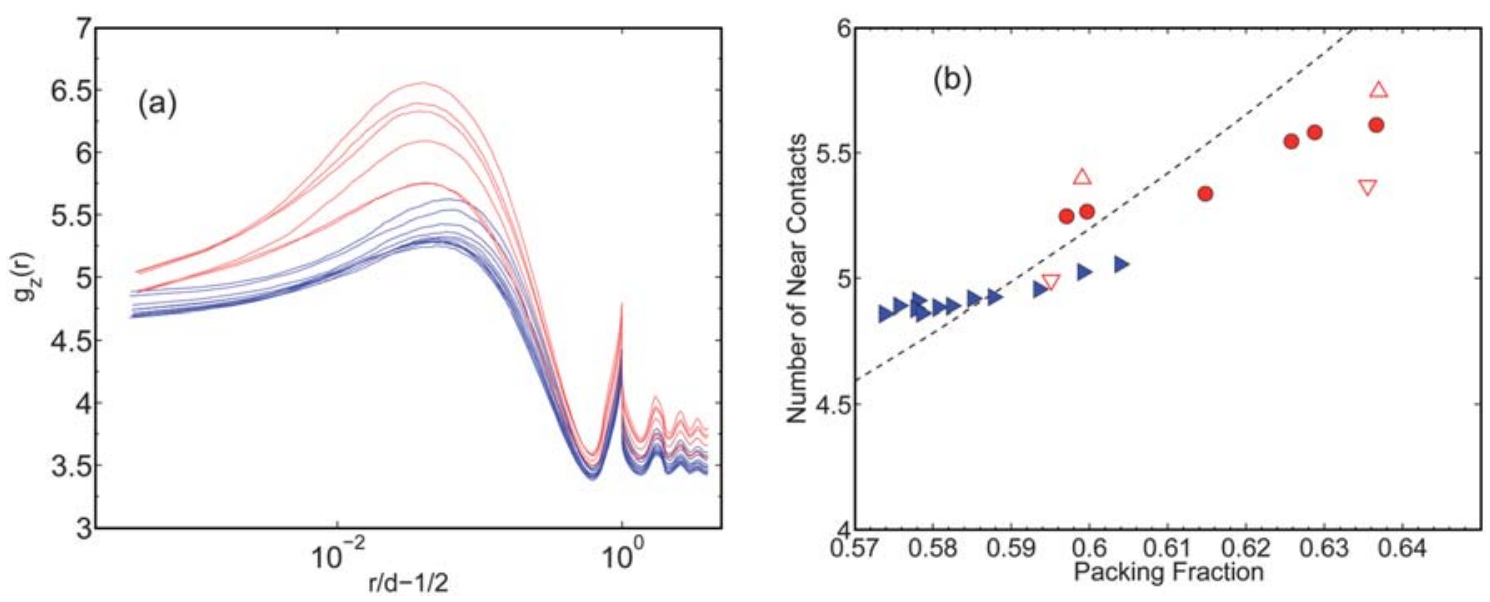

Fig. 8 (Left, a) Modified radial distribution function $g_{z}(r)$ (see text). The six higher curves above refer to the acrylic bead system whereas the 12 lower curves below concern the fluidized bed systems. (Right, b) Number of near contacts estimated from the value of $g_{z}(r)$ at $r-d / 2=0.004 d$. The dashed line is $z=2 \sqrt{3} \Phi /(1-\Phi){ }^{44}$ As in the previous figures, the triangles ' $\triangle$ ' refer to fluidized bed samples and the circles ' $O$ ' to the dry acrylic bead samples; the symbols $\nabla$ refer to two samples (B and F) relaxed with a larger friction coefficient ( $\mu=0.9$ instead of 0.28 ); the symbols $\Delta$ refer to samples $\mathrm{B}$ and $\mathrm{F}$ relaxed with a smaller friction coefficient $(\mu=0.2)$. 
Fig. $8 \mathrm{~b}$ this estimation of the number of near contacts is shown as function of the sample packing fraction. In Table 2 the values are reported together with the maximal values of $g_{z}(r)$ in the proximity of $r / 2$.

Based on these results, it appears that aside from the number of actual contacts $z_{c}$, which is a well defined quantity, there isn't any clear instrument to unequivocally identify the near neighbors which are relevant to physical properties of the packing. From a geometrical perspective this is an ill-posed problem because there isn't any natural cut-off distance beside the sphere sizes. However, from a dynamical perspective there might be a "region of influence" where neighboring spheres might become in contact with the central one when the system is infinitesimally perturbed. In principle, this can be tested with simulations and will be the topic of a future work.

\section{B. Comparison with deconvolution method}

In a previous paper ${ }^{9}$ the average number of grains in contact was estimated by means of a deconvolution method using an error function to fit the experimental $z(r)$ in the region $r<d$ and assuming that the observed smooth increase is due to the grain
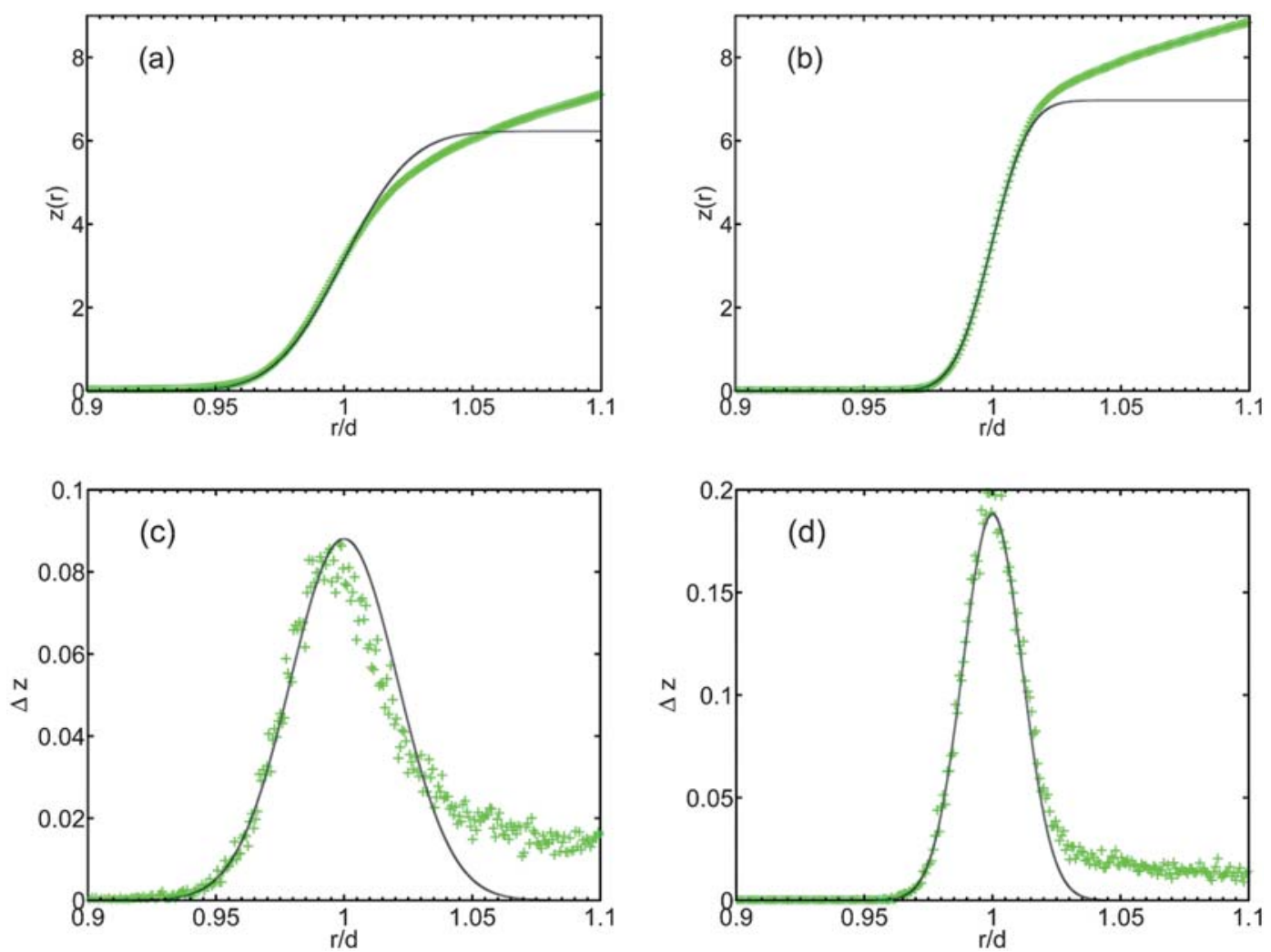

Fig. 9 Two examples for samples FB18 (a,c) and F (b,d) of the estimation of the number of spheres in contact by means of the deconvolution method. In $(\mathrm{a}, \mathrm{b})$ the symbols ' + ' are the values of $z(r)$ from XCT experimental data and the lines are the error functions that best fit the data. In (c,d) the symbols ' + ' report the differences $z(r+\delta r)-z(r)$ with $\delta r=0.000743 d$ as measured from XCT experimental data. The lines are the normal distributions that are best fitting the data for $r<d$.

Table 2 Estimation of the neighbors in contact $\left(z_{D E}\right)$ computed by means of the deconvolution method (see Table 1 in ref. 9). Number of near neighbors computed from $g_{z}(r)$ at $r-d / 2=0.004 d$ and from the maximum of $g_{z}(r)$. The number of actual contacts $z_{c}$ are also reported for comparison

\begin{tabular}{lllllllll}
\hline Sample & $z_{D E}$ & $g_{z}(0.004 d+d / 2)$ & $\max \left(g_{z}(r)\right)$ & $z_{c}$ & Sample & $z_{D E}$ & $g_{z}(0.004 d+d / 2)$ & $\max \left(g_{z}(r)\right)$ \\
\hline FB14 & 5.60 & 4.91 & 5.29 & 4.70 & $\mathrm{~A}$ & 5.81 & 5.26 & 5.25 \\
FB15 & 5.70 & 4.88 & 5.28 & 4.71 & $\mathrm{~B}$ & 5.91 & 5.25 & 5.75 \\
FB16 & 5.30 & 4.88 & 5.30 & 4.71 & $\mathrm{C}$ & 6.77 & 5.34 & 4.80 \\
FB17 & 5.40 & 4.89 & 5.29 & 4.69 & $\mathrm{D}$ & 6.78 & 5.55 & 6.09 \\
FB18 & 5.00 & 4.86 & 5.25 & 4.70 & $\mathrm{E}$ & 6.95 & 5.58 & 6.33 \\
FB19 & 5.30 & 4.89 & 5.31 & 4.72 & $\mathrm{~F}$ & 6.97 & 5.61 & 6.39 \\
FB20 & 5.80 & 4.86 & 5.29 & 4.69 & & & 5.05 \\
FB21 & 5.80 & 4.92 & 5.32 & 4.74 & & & \\
FB22 & 5.70 & 4.92 & 5.36 & 4.75 & & & \\
FB23 & 5.90 & 4.96 & 5.43 & 4.78 & & & & \\
FB24 & 5.90 & 5.06 & 5.63 & 4.89 & & & & \\
FB27 & 6.00 & 5.02 & 5.54 & 4.86 & & & & \\
\hline
\end{tabular}


polydispersity. Fig. 9 shows that this method captures well the typical distribution of distances in the region $r<d$. However, from Table 2, where the values of the number of contacts per sphere computed by means of this deconvolution method $\left(z_{D E}\right)$ are compared with $z_{c}$, it is evident that this method largely overestimates the number of actual contacts. This is probably due to the fact that in the presence of polydispersity grains that are not in contact can stay at a relative distance smaller than $d$. Interestingly the numbers retrieved in ref. 9 were in good agreement with several experimental estimations ${ }^{46,47}$ pointing out the need for better experimental methods to directly access this quantity.

\section{Local relation between packing fraction and number of contacts}

We have seen that the average number of contacts $z_{c}$ for the whole packing increases almost linearly with the packing fraction. In a previous paper ${ }^{56}$ it was pointed out that, at the grain scale, the number of contacts and the packing fraction are also locally related. Fig. 10 reports the average number of contacts $\langle z\rangle$ for spheres at a given local packing fraction $\phi$. The local packing fraction $\phi$ is calculated by computing the fraction between the volume of a sphere $V_{s}=\pi d^{3} / 6$ and the volume $V$ of the Voronoi cell around the sphere, i.e. $\phi=V_{s} / V$. Note that the global packing fraction $(\Phi)$ is retrieved similarly from $\Phi=V_{s} /\langle V\rangle$. Let us stress that the quantities reported in Fig. 10 are different from the ones in Fig. 7, indeed Fig. 10 refers to a local relation and not to the relation for the whole packing.

An analytical approach in ref. 44 proposes the following relation:

$$
\langle z\rangle=2 \sqrt{3} \frac{\phi}{1-\phi}
$$

In Fig. 10 this analytical prediction is compared with the data for all the fluidized beads and dry samples. One can see that significant deviations are observed. Note that the large error bars in the figures are not reporting an uncertainty on the measure, which is exact in this case, but are visually showing the spread of the distribution of the contact number per sphere at a given local packing fraction. We discuss such a distribution in detail in Sec. 9.

\section{Comparison with isostatic condition}

The samples considered here are in a mechanically stable state. This applies to both the experimental samples that were measured at rest and to the DEM relaxed samples that were allowed a sufficiently long time to achieve the dynamical arrest of all grains. From a simple counting of the number of degrees of freedom with respect to the number of constraints one can infer the minimum expected average number of contacts per grains that can allow mechanical stability. Samples exactly satisfying such a counting are generally referred to as being at the isostatic condition. One must stress that it has been mathematically proven that such a counting does not provide either a sufficient or a necessary condition for stability. ${ }^{21}$ Nonetheless, the isostatic condition gives a good indication of the approximate value of contacts above which one can reasonably expect to find mechanically stable packings.

In our samples we have frictional spheres with rotational degrees of freedom that interact with both normal and tangential forces. The forces are the free variables of the problem that are subject to the constraints of zero total force and zero total torque acting on each grain. Normal forces have directions determined by the position of the grains, and therefore they account for only one scalar variable per contact, counting to:

$$
N_{n}=z_{c} \frac{N}{2}
$$

Tangential forces act in the plane between the two spheres, in general they have $D-1$ components (here $D=3$ is the space dimensionality). However, some contacts can be at the slipping threshold where $F_{t}=\mu F_{n}$ and they cannot hold any extra tangential load in that direction, in this case the number of independent components is reduced by one becoming $D-2=1$. Overall the total number of variables associated with tangential forces is:

$$
N_{t}=z_{c} \frac{N}{2}[(D-1)(1-q)+(D-2) q]=z_{c} \frac{N}{2}(D-1-q)
$$

where $q$ is the fraction of contacts at the slipping threshold that cannot hold any extra tangential load, conversely $1-q$ is the fraction of contacts that are below the slipping threshold. The force balance must be satisfied for each grain and for each
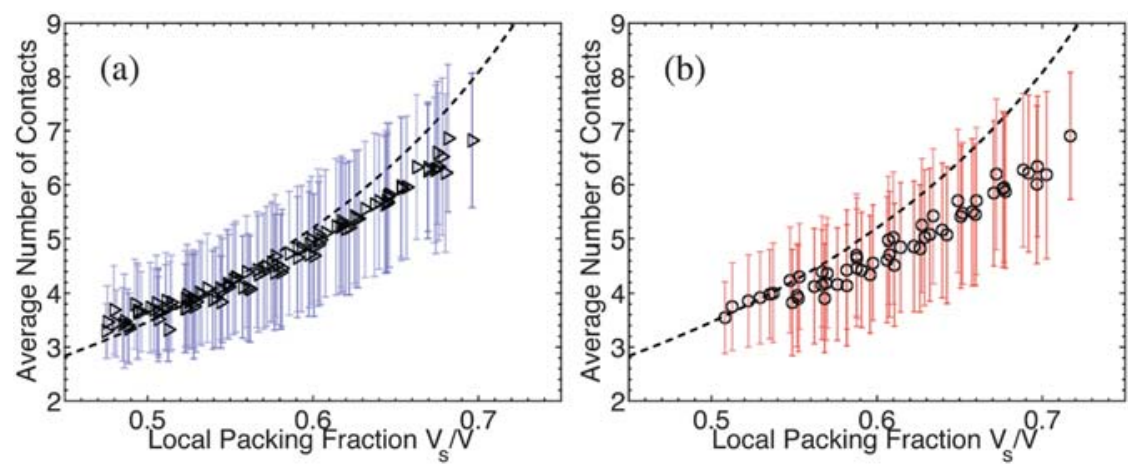

Fig. 10 Local relation between the average number of neighbors in contact $\langle z\rangle$ and the local packing fraction $\phi=V_{s} / V$ for (a) the fluidized bed samples and (b) the dry acrylic beads samples. The bars report the standard deviation of the distribution $\sigma_{z}=\sqrt{\left\langle z^{2}\right\rangle-\langle z\rangle^{2}}$. The dashed line is the theoretical behavior from: ${ }^{44}\langle z\rangle=2 \sqrt{3} /(1 / \phi-1)$. Volumes are binned into 24 discrete equal intervals. The choice of binning has very marginal effects. 
component of the force acting on the center of mass, and thus the number of equations for force balance is:

$$
E_{f}=D N
$$

Whereas the number of equations for torque balance is:

$$
E_{t}=\frac{D(D-1)}{2} N
$$

The balance between number of variables and number of equations is:

$$
N_{n}+N_{t}=E_{f}+E_{t}
$$

resulting in the following condition for the average number of contact per grain at the 'isostatic limit':

$$
z_{\text {ISO }}=\frac{D(D+1)}{D-q}=\frac{12}{3-q}
$$

It is clear that this implies $4 \leq z_{\text {ISO }} \leq 6$ depending on the fraction $q$ of contacts that are at the slipping threshold. This number can be computed from the DEM relaxed samples and the values of $q$ for all the samples are reported in Table 1 . We observe that there is a sizable fraction of contacts at the slipping threshold with a larger fraction (about 15\%) in the dry bead samples with respect to the fraction in the fluidized beds samples (about 3\%). This is due to the larger value of the friction coefficient in the fluidized bed samples. It is indeed clear that $q$ should depend on friction with the limit $q \rightarrow 1$ associated with the limiting case of infinitesimally small friction coefficients and conversely the limit $q \rightarrow 0$ associated with the limiting case of very large friction coefficients. Overall we see from Table 1 that all samples are 'hyperstatic' with values of $z_{c}$ always considerably larger than the corresponding $z_{I S O}$. Let us note that infinitesimal variations might change the state of a contact at the slipping threshold into a non-slipping one and vice versa. However, the average number of contacts at the slipping threshold turns out to be a well defined quantity which is robust to infinitesimal perturbation of the system. This has been tested numerically by following the evolution of $q$ over the whole simulation time. We indeed observed that $q$ reaches values which are within $1 \%$ of the final measured value after about half of the simulation time, well before the packing has reached dynamical arrest.

In the previous calculation for the isostatic limit we ignored the effect of the boundaries. On the other hand, boundaries are essential to hold the structure in place and cannot be ignored. We will expand on this issue in the next section.

\section{A. Critical cluster size}

In these packings, mechanical stability is induced by the boundaries that literally hold all the sample in place. Let us now imagine to extract an internal cluster of spheres and investigate whether it can be mechanically stable under compression and/or shearing occurring at its boundaries. In this case, only the contacts between the grains inside the cluster contribute to stability. For such a cluster, the average number of internal-internal contacts per grain is smaller than $z_{c}$ because one must subtract the contacts with grains external to the cluster. For large clusters this number will converge towards $z_{c}$ but for small clusters it could be considerably lower than $z_{c}$ and even become smaller than $z_{I S O}$. The mechanical stability of sub-parts of the packing can be inferred by dividing the entire sample in a grid of cubic cells of edge-size $l$ and looking at the average internal-internal number of contacts inside each of these cells. The smallest cluster size $l^{*}$ where the average number of internal-internal contacts per grain is larger than or equal to $z_{I S O}$, can be considered as the 'critical' size. Table 1 reports the critical sizes $l^{*}$ for all samples. As one can see they vary in a range between 9 to 12 sphere diameters. This critical size is an estimate of the size of the smaller cluster which might still act as a rigid body upon external perturbations, below this size the system is likely to be locally in a mechanically unstable state and, in the sample, it is actually held in place by the presence of static neighbors. As mentioned before, the isostatic condition is neither a sufficient nor a necessary condition for stability ${ }^{21}$ and therefore this criteria is only providing an indication for the critical size. A precise formulation of the problem, requires the complete analysis of the stability problem $^{57,58}$ but this goes beyond the purpose of the present paper.

\section{Distribution of the number of contacts}

The average number of neighbours in contact with a given grain is an important quantity. However, from a grain perspective, we

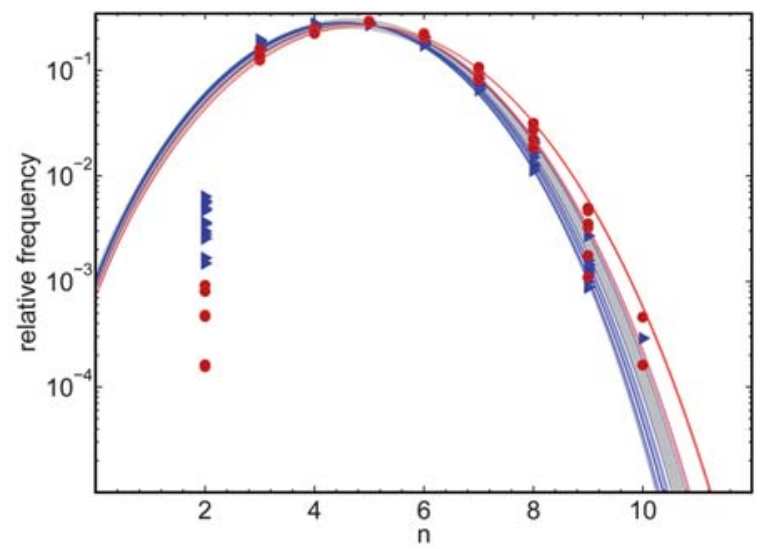

Fig. 11 (Left) Distribution of the neighbors in contact for all the experimental samples: fluidized bed $(\triangleright)$ and dry acrylic beads $(O)$. The line is the function in eqn (16) with best fitting values for the parameter $z^{*}$. (Right) Same plot shown on a semi-log scale. 

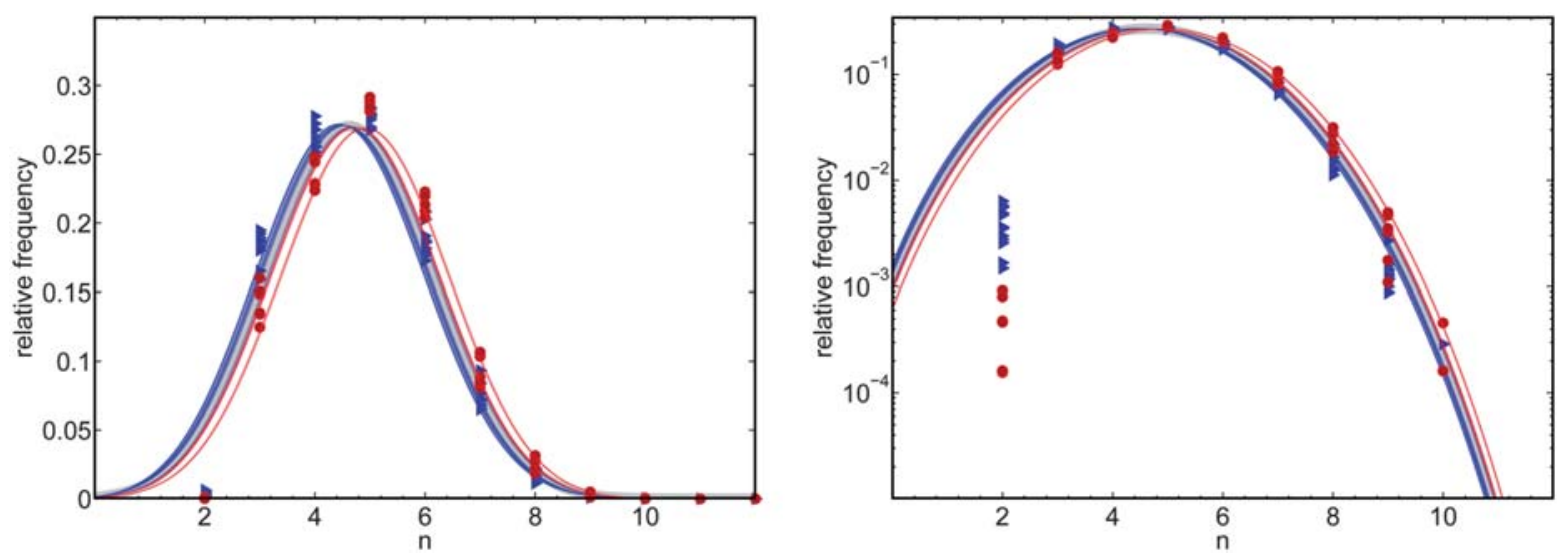

Fig. 12 (Left) Distribution of the neighbors in contact for all the experimental samples: fluidized bed ( $\triangleright$ ) and dry acrylic beads $(\bigcirc)$. The lines are the function in eqn (17) with $z^{*}=4 /(2-\sqrt{3}) \sim 14.9$ and the best fitting values of $\lambda$. (Right) Same plot shown on a semi-log scale.

see that spheres are in contact with variable numbers of neighbors with a probability that follows a distribution which is reported, for all samples, in Fig. 11 and 12. As one can see, these distributions are "bell-shaped" with a number of contacts that range from a minimum of 2 to a maximum of 12 . The problem that we briefly address in this section is the statistical mechanics mechanism that might be at the origin of this distribution.

Let us here introduce a simple 'free volume theory' which is formally similar to the free volume theory for a gas in a given volume $^{59}$ but applied to the spheres in contact with a given sphere. Let us consider the total solid angle around a sphere $4 \pi$ as a 'volume' which is locally filled by the neighboring spheres each occupying a solid angle $\Omega$. In such a system, if we have $z$ spheres in contact, each sphere has access to a maximum portion of the solid angle equal to $4 \pi-z \Omega$. In the 'microcanonical' ensemble the partition function of a 'gas' of $z$ of such particles is $W(z)=(4 \pi-z \Omega)^{z} z$ ! . The probability to have a configuration with $z$ neighbors is therefore consistently $p(z)=W(z) / \sum_{z^{\prime}} W\left(z^{\prime}\right)$ which can be written as:

$$
p(z)=\frac{c}{\Gamma(z+1)}\left(4 \pi \frac{z^{*}-z}{z^{*}}\right)^{z}
$$

where we introduced the symbol $z^{*}=4 \pi / \Omega$, which is the upper bound for the number contacts, and $c$ is a renormalization constant such that $\sum_{z} p(z)=1$. In Fig. 11 the distribution of the number of neighbors in contact for all the samples are reported. The lines are the plot of eqn (16) where the best fits for $z^{*}$ are used. The resulting best fit values are in a narrow range between 14 and 15 . As one can see, the theoretical prediction is very satisfactory, describing well the overall behavior except for the probability for $z=2$ which is smaller than expected from eqn (16). This is not surprising as the theory ignores mechanical stability. In our systems, that include friction, two frictional contacts can be sufficient to hold a grain in place. However, it is rather unlikely that grains spontaneously jam in these configurations, and our simple theory over-estimates their occurrence in real packings.

The use of $z^{*}$ as a free fitting parameter is reasonable given that different packing strategies affect differently the likelihood of filling the solid angle around a sphere. However, in a strict mathematical sense, the free volume occupied by a touching sphere is $\Omega=2 \pi[1-\cos (\pi / 6)]=\pi(2-\sqrt{3})$ and therefore $z^{*}=4 /(2-\sqrt{3}) \sim 14.9$. An alternative approach is considering $z^{*}$ fixed and instead taking into account the different packing characteristics by using a sort of 'chemical potential' that gives a weight for the likelihood for a sphere to come in contact with another sphere. This alternative framework is equivalent to the 'grand canonical' ensemble (at $\beta=0$ ) and the 'chemical potential' is a Lagrange multiplier $\lambda$ that controls the average number of neighbors in contact. The expression for the probability becomes:

$$
p(z)=\frac{c}{\Gamma(z+1)}\left(4 \pi \frac{z^{*}-z}{z^{*}}\right)^{z} \exp (-\lambda z)
$$

where $c$ is a renormalization constant such that $\sum_{z} p(z)=1$ and, in this case, $z^{*}=4 /(2-\sqrt{3})$. Fig. 12 shows that this alternative approach is also very effective in describing the distribution of the number of neighbors. The estimated values of $\lambda$ vary in a range between 0.12 to 0.31 following an almost linear decreasing trend with packing fraction.

\section{Distribution of forces and pressure}

Understanding how stress distributes in these systems is of great relevance to unveil the mechanisms underneath mechanical stability and the jamming transition in these complex materials.

Let us first note that the topological properties of these samples are rather insensitive to the relative depth of the grains in the sample. An example is given in Fig. 13 where the average number of neighbors and the standard deviation of the distribution are plotted for several layers at different depths, showing that these quantities rest almost unchanged. A different behavior is instead observed for the average normal force in each layer. At increasing depths, the force increases due to the larger weight of the grains above. We take this effect into account in the following discussion.

Fig. 14 reports the distribution of the normal forces between grains measured in an internal part of the sample 4 sphere diameters away from the boundaries. We observe that a large fraction of contacts are carrying small forces and conversely a small fraction of contacts carry large forces. When plotted in 

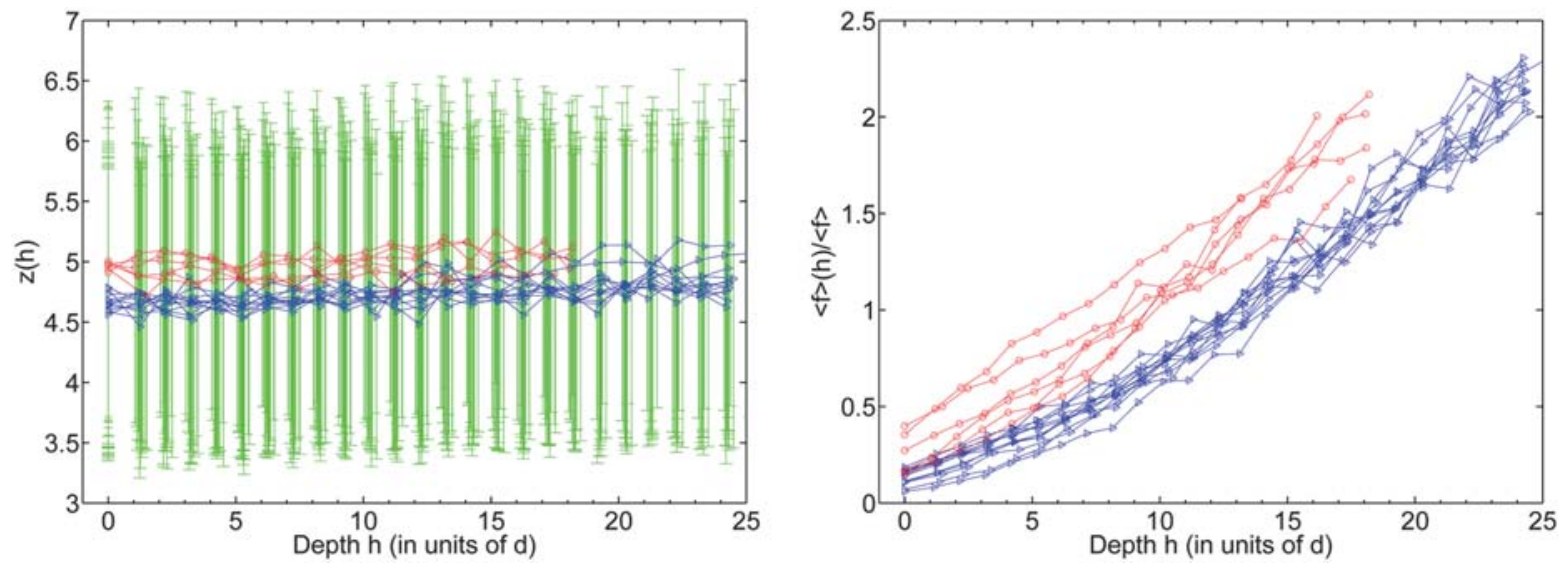

Fig. 13 (Left) Average number of contacts per grain vs. depth. The error bars represent the standard deviation. (Right) Average rescaled force vs. depth. Symbols: triangles ' $\triangleright$ ' fluidized bed samples; circles ' $O$ ' dry acrylic bead samples.
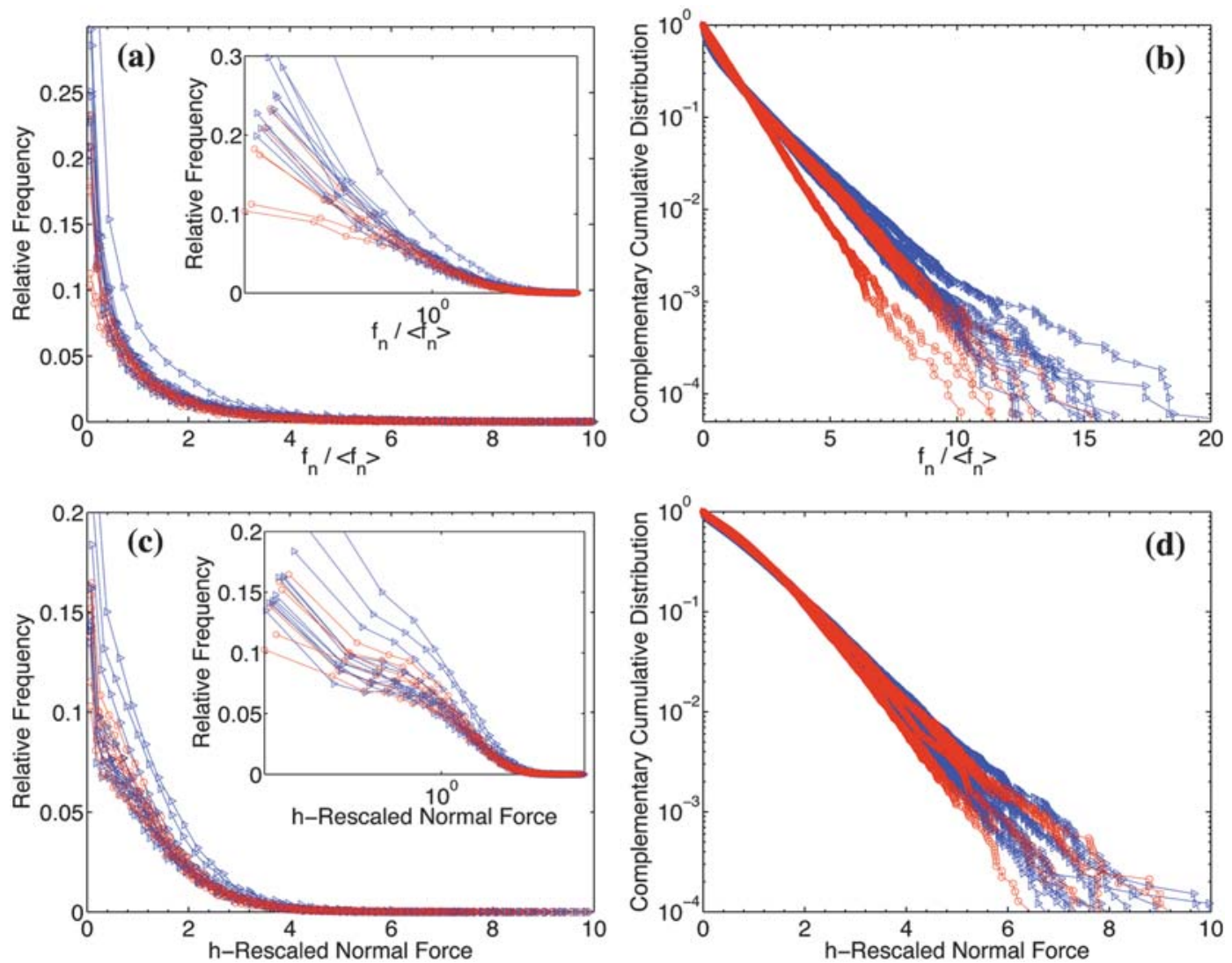

Fig. 14 Distribution of normal forces inside the sample. (Left, a,c) Frequency distributions, the insets have linear-log scales to visually expand the region of small forces; (right, b,d) complementary cumulative distributions in log-linear scales. (Above, a,b) Distributions of the forces rescaled by the average force in the sample. (Below, c,d) Distributions of the forces rescaled by the average force in each layer at different depths. Symbols: triangles ' $\triangleright$ ' fluidized bed samples; circles ' $O$ ' dry acrylic bead samples.

log-linear scale the distributions reveal a linear behavior in their tails indicating an exponential decay. We observe that the rescaling of the force with the mean force measured in a layer at the corresponding height (the one reported in Fig. 13) results in a cleaner gathering into a consistent linear behavior in the tail region of all distributions. On the other hand, the rescaling seems to affect the small forces part where the behaviors of the plots for each sample become less homogeneous with the appearance of a change in the curvature.

\section{A. Local forces acting on a sphere}

In Fig. 15 we plot the average of the arithmetic sum of the moduli of all normal forces acting on a sphere with a given number of 

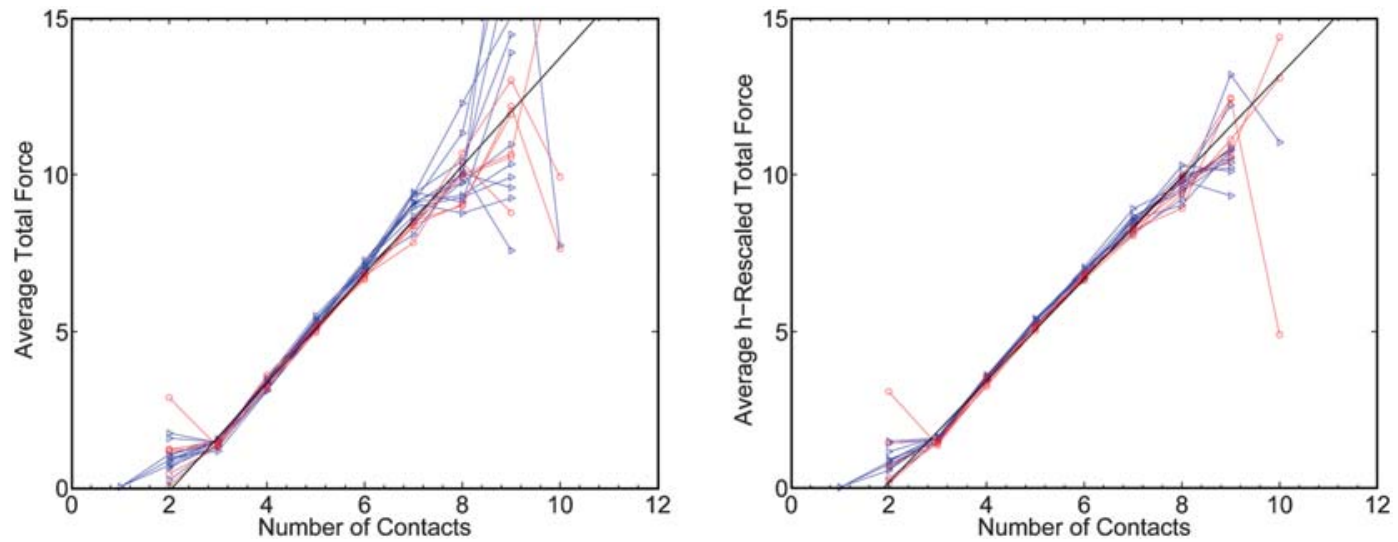

Fig. 15 Average of the arithmetic sum of the moduli of all normal forces (total force) acting on a sphere with a given number of contacts $z v s$. number of contacts $z$. (Left) The total force is rescaled to the sample average. (Right) The total force is rescaled by dividing the force by the mean force in the layer at the grain height (Fig. 13). Symbols: triangles ' $\triangleright$ ' fluidized bed samples; circles ' $O$ ' dry acrylic bead samples.

contacts $z$. We observe a very neat linear increase with $z$. Note that a linear trend would be expected if the forces at each contact were independent on the forces at the other contacts of a given sphere.

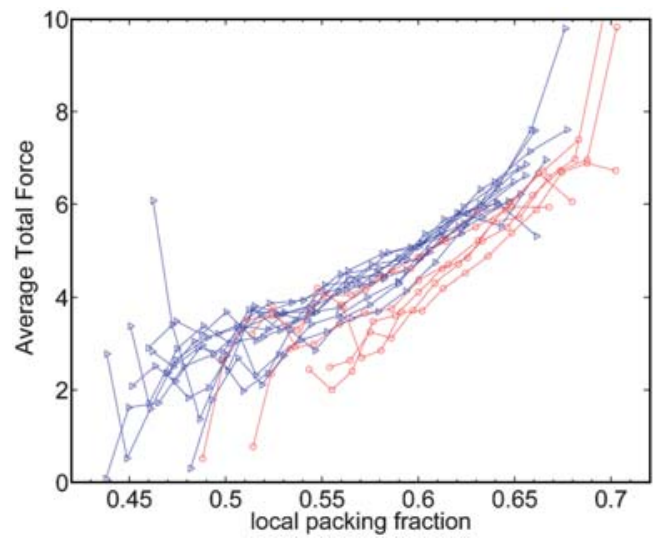

In Fig. 16 we report the average of the arithmetic sum of the moduli of all normal forces (total force) acting on a sphere with a given local packing fraction i.e. $\phi=V_{s} / V$. The figure reports two rescalings with respect to the average force per contact in the

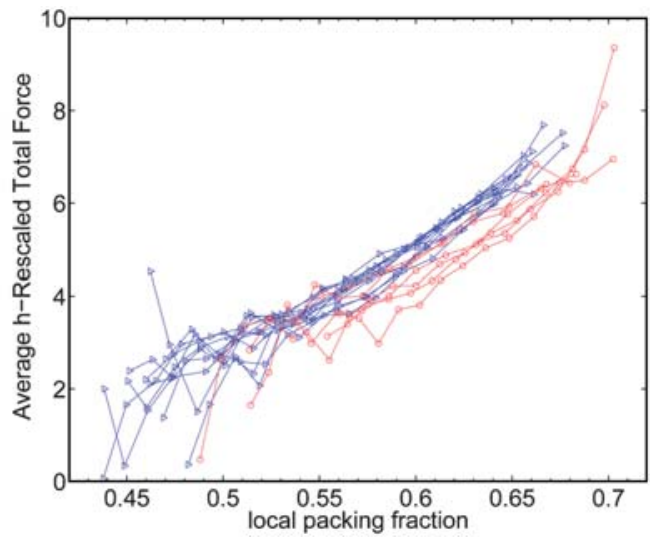

Fig. 16 Average of the arithmetic sum of the moduli of all normal forces (total force) acting on a sphere with a given local packing fraction vs. local packing fraction. (Left) The total force is rescaled to the sample average force per contact. (Right) The total force is rescaled by dividing the force by the mean force per contact in the layer at the grain height (Fig. 13). Symbols: triangles ' $\square$ ' fluidized bed samples; circles ' $O$ ' dry acrylic bead samples.
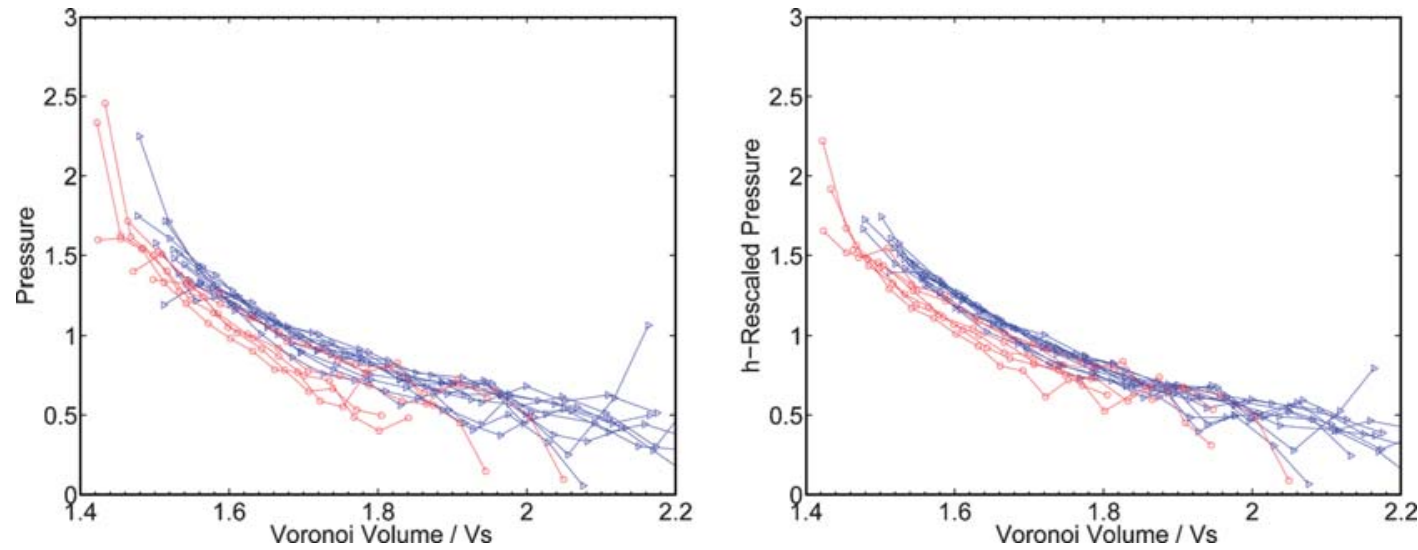

Fig. 17 Average pressure (average of the arithmetic sum of the moduli of all normal forces divided by Voronoi area) acting on a sphere with a given Voronoi volume $v s$. Voronoi volume divided by the volume of the sphere $V_{s}$. (Left) The pressure is rescaled by dividing by the mean force per contact in the whole sample. (Right) The pressure is rescaled by dividing by the mean force per contact in the layer at the grain height (Fig. 13). Symbols: triangles ' $\triangleright$ ' fluidized bed samples; circles ' $O$ ' dry acrylic bead samples. 
whole sample and in each layer. It is clear that there is a monotonic relation with more densely packed grains carrying larger forces. This means that larger Voronoi cells are carrying smaller forces. This is consistent with the previous findings (Fig. 15) because, indeed, larger Voronoi cells have fewer neighbors than smaller ones (as discussed in Sec. 7 and reported in Fig. 10).

\section{B. Local pressure}

In Fig. 17 we finally report a similar measure, where instead of the force the 'pressure' is considered. In this context the pressure is defined as the average of the arithmetic sum of the moduli of all normal forces divided by the total surface area of the corresponding Voronoi cell. Data are renormalized by dividing by the average force (either in the whole sample or in the layers) and by multiplying by $d^{2}$ (to renormalize to the case of unit diameter spheres). The result is analogous to what is observed for the forces, with the smaller Voronoi volumes (larger $\phi$ ) carrying larger pressures.

\section{Conclusions and outlook}

In this work we have presented results obtained from a newly developed 'virtual laboratory' platform which combines direct experimental imagining data from XCT with DEM simulation tools. We have shown that such a combination is very powerful, providing 'virtual samples' which are geometrically virtually identical to the experimental ones but from which we can access physical quantities that would not be easily obtainable from experiments alone. For instance, we have been able to greatly improve our estimate of the number of grains in contact and to study accurately the region of near contacts. In particular, we pointed out that the exponential law for the growth in the number of near neighbors with the radial distance is well followed across all samples but the exponent is not universal, varying from 0.7 at low packing fractions to 0.5 for the densest sample at $\Phi \simeq 0.64$. We have discussed how close our packings are to isostaticity and how a critical size of around ten grain diameters emerges for minimal clusters satisfying the isostatic condition. The relation between average number of contacts and packing fraction has been investigated both globally and locally and it has been compared with analytical predictions. The distribution of the number of contacts per grain has been investigated and we introduced a simple meaningful model based on the idea of free 'particles' sharing the solid angle around a sphere which turns out to reproduce remarkably well the observed distributions. We have been able to estimate the complete set of forces on each individual grain and we have shown that the normal forces follow exponential distributions. We have also measured the local pressure on each Voronoi cell and we have uncovered a linear relation between the sum of the normal forces and the number of contacts on each bead.

These results are a demonstration of the great potential of our approach. We are able to utilize real experimental data obtained from granular packings as our starting point, and investigate the relationship between the internal geometric properties and the nature of the inter-grain force network. This approach can provide the key bridge between the two main methodologies, experimental and computational, that are being used to explore and better understand granular matter. By more closely bringing together these investigations, we can obtain a more complete and confident picture of the properties of granular matter; providing the key information necessary to formulate and test new theories.

In future work we will expand on this, examining the dynamic properties of packings, considering systems under external loadings and under shear, ${ }^{60}$ and examining properties of packings at the point of failure. ${ }^{61}$ We will also explore the combination of high speed tomography and other bulk imaging techniques with DEM during dense granular flow and at the jamming transition. The role of grain shape in granular systems is also of great interest ${ }^{62-66}$ and an extension of this technique to consider three-dimensional non-spherical grains will also be pursued.

\section{Acknowledgements}

We wish to thank the referees for their very careful and precise scrutiny of the first draft of this manuscript. Their suggestions have been very important and greatly help to improved the paper. Many thanks to T. J. Senden and M. Saadatfar for the tomographic data. This work was partially supported by the Australian Partnership for Advanced Computing National Facilities (APAC).

\section{References}

1 L. P. Kadanoff, Rev. Mod. Phys., 1999, 71, 435.

2 H. M. Jaeger, S. R. Nagel and R. P. Behringer, Rev. Mod. Phys., 1996, 68, 1259.

3 C. S. Campbell, Annu. Rev. Fluid Mech., 1990, 22, 57.

4 G. T. Herman, Fundamentals of computerized tomography: Image reconstruction from projection, Springer-Verlag, Berlin, 2009, 2nd edn.

5 J. Keeler, Understanding NMR Spectroscopy, John Wiley \& Sons, New York, 2005.

6 J. Brujić, S. F. Edwards, I. Hopkingson and H. A. Makse, Phys. A, 2003, 327, 201.

7 M. Cui and R. Adrian, Exp. Fluids, 1997, 22, 261.

8 T. S. Majmudar and R. P. Behringer, Nature, 2005, 435, 1079.

9 T. Aste, M. Saadatfar and T. J. Senden, Phys. Rev. E: Stat., Nonlinear, Soft Matter Phys., 2005, 71, 061302.

10 M. Tsukahara, S. Mitrovic, V. Gajdosik, G. Margaritondo, L. Pournin, M. Ramaioli, D. Sage, Y. Hwu, M. Unser and T. M. Liebling, Phys. Rev. E: Stat., Nonlinear, Soft Matter Phys., 2008, 77, 061306.

11 G. Delaney, S. Inagaki, and T. Aste, in Granular and Complex Materials, ed. T. Aste, T. D. Matteo, and A. Tordesillas, World Scientific, 2007, vol. 8, pp. 169-185.

12 G. W. Delaney, S. Inagaki and T. Di Matteo, Proc. SPIE, 2008, vol. 6802, 68020G.

13 A. Mehta, G. C. Barker and J. M. Luck, Proc. Natl. Acad. Sci. U. S. A., 2008, 105, 8244 .

14 M. Nicodemi, A. Coniglio and H. J. Herrmann, Phys. Rev. E: Stat. Phys., Plasmas, Fluids, Relat. Interdiscip. Top., 1997, 55, 3962.

15 C. S. O'Hern, L. E. Silbert, A. J. Liu and S. R. Nagel, Phys. Rev. E: Stat., Nonlinear, Soft Matter Phys., 2003, 68, 011306.

16 H. A. Makse, N. Gland, D. L. Johnson and L. Schwartz, Phys. Rev. E: Stat., Nonlinear, Soft Matter Phys., 2004, 70, 061302.

17 H. G. Matuttis, S. Luding and H. J. Herrmann, Powder Technol, 2000, 109, 278.

18 T. Pöschel and T. Schwager, Computational Granular Dynamics, Springer-Verlag, Berlin, 2005.

19 P. A. Cundall and O. D. L. Strack, Geotechnique, 1979, 29, 47.

20 S. Hutzler, G. Delaney, D. Weaire and F. MacLeod, Am. J. Phys, 2004, 72, 1508.

21 T. Aste and D. Weaire, The Pursuit of Perfect Packing, Institute of Physics, Bristol, 2000. 
22 N. V. Brilliantov, F. Spahn, J. Hertzsch and T. Pschel, Phys. Rev. E. Stat. Phys., Plasmas, Fluids, Relat. Interdiscip. Top., 1996, 53, 5382.

23 T. S. Majmudar, M. Sperl, S. Luding and R. P. Behringer, Phys. Rev. Lett., 2007, 98, 058001.

24 See, http://wwwrsphysse.anu.edu.au/granularmatter/(2006-2009).

25 T. Aste, J. Phys.: Condens. Matter, 2005, 17, S2361.

26 T. Aste, T. Di Matteo, M. Saadatfar, T. J. Senden, M. Schröter and H. L. Swinney, Europhys. Lett., 2007, 79, 24003, 1.

27 M. Jerkins, M. Schröter, H. L. Swinney, T. J. Senden, M. Saadatfar and T. Aste, Phys. Rev. Lett., 2008, 101, 018301.

28 T. Aste, M. Saadatfar, A. Sakellariou and T. J. Senden, Phys. A, 2004, $339,16$.

29 M. Schröter, D. I. Goldman and H. L. Swinney, Phys. Rev. E: Stat., Nonlinear, Soft Matter Phys., 2005, 71, 030301.

30 A. Sakellariou, T. J. Senden, T. J. Sawkins, M. A. Knackstedt, A. Limaye, C. H. Arns, A. P. Sheppard, and R. M. Sok, in Proceedings of SPIE, ed. U. Bonse, 2004, vol. 5535, pp. 166-171.

31 A. Sakellariou, T. J. Sawkins, T. J. Senden and A. Limaye, Phys. A, 2004, 339, 152.

$32 \mathrm{~S}$. Beucher and C. Lantuéjoul, Use of watersheds in contour detection in International workshop on image processing, real-time edge and motion detection/estimation, Rennes, France, 1979, pp. 2.1-2.12.

33 K. Johnson, Contact Mechanics, Cambridge University Press, Cambridge, 1985.

34 L. Landau and E. Lifshitz, Theory of Elasticity, Pergamon, New York, 1970.

35 H. A. Makse, N. Gland, D. L. Johnson and L. Schwartz, Phys. Rev. E: Stat., Nonlinear, Soft Matter Phys., 2004, 70, 061302.

36 C. Goldenberg and I. Goldhirsch, Phys. Rev. E: Stat., Nonlinear, Soft Matter Phys., 2008, 77, 041303.

37 C. H. Rycroft, M. Z. Bazant, G. S. Grest and J. W. Landry, Phys. Rev. E: Stat., Nonlinear, Soft Matter Phys., 2006, 73, 051306.

38 J. Schafer, S. Dippel and D. Wolf, J. Phys. I, 1996, 6, 5.

39 L. Brendel and S. Dippel, Physics of Dry Granular Media, 1998, 39.

40 A. Donev, S. Torquato, F. H. Stillinger and R. Connelly, Phys. Rev. E: Stat., Nonlinear, Soft Matter Phys., 2005, 71, 011105.

41 R. D. Kamien and A. J. Liu, Phys. Rev. Lett., 2007, 99, 155501, pages 4.

42 R. P. Ojha, P. A. Lemieux, P. K. Dixon, A. J. Liu and D. J. Durian, Nature, 2004, 427, 521.

43 L. E. Silbert, A. J. Liu and S. R. Nagel1, Phys. Rev. E: Stat., Nonlinear, Soft Matter Phys., 2006, 73, 041304.

44 C. Song, P. Wang and H. A. Makse, Nature, 2008, 453, 629.

45 W. O. Smith, P. D. Foote and P. F. Busang, Phys. Rev., 1929, 34, 1271.

46 J. D. Bernal and J. Mason, Nature, 1960, 188, 910.

47 G. D. Scott, Nature, 1962, 194, 956.

48 G. W. Delaney, D. Weaire and S. Hutzler, Europhys. Lett., 2005, 72, 990.
49 G. Mason and W. Clark, Nature, 1966, 211, 957.

50 G. Mason, Nature, 1968, 217, 733.

51 P. J. Steinhardt, D. R. Nelson and M. Ronchetti, Phys. Rev. B: Condens. Matter, 1983, 28, 784.

52 G. T. Seidler, G. Martinez, L. H. Seeley, K. H. Kim, E. A. Behne, S. Zaranek, B. D. Chapman, S. M. Heald and D. L. Brewe, Phys. Rev. E: Stat. Phys., Plasmas, Fluids, Relat. Interdiscip. Top., 2000, 62, 8175 .

53 A. Sederman, P. Alexander and L. Gladden, Powder Technol., 2001, $117,255$.

54 S. Torquato and F. Stillinger, J. Phys. Chem. B, 2001, 105, 11849

55 L. E. Silbert, D. Ertas, G. S. Grest, T. C. Halsey and D. Levine, Phys. Rev. E: Stat., Nonlinear, Soft Matter Phys., 2002, 65, 031304.

56 T. Aste, M. Saadatfar and T. J. Senden, J. Stat. Mech.: Theory Exp., 2006, P07010.

57 R. M. Baram, H. J. Herrmann and N. Rivier, Phys. Rev. Lett., 2004, 92, 044301.

58 N. Rivier, ISSN 0022-3093, proceedings of the 5th International Discussion Meeting on Relaxations in Complex Systems, 5th International Discussion Meeting on Relaxations in Complex Systems, J. Non-Cryst. Solids, 2006, 352, 4505.

59 T. Hill, Statistical mechanics, Mc Graw-Hill, New York, 1956.

60 M. E. Cates, J. P. Wittmer, J. Bouchaud and P. Claudin, Phys. Rev. Lett., 1998, 81, 1841.

61 P. R. Welker and S. C. McNamara, Phys. Rev. E: Stat., Nonlinear, Soft Matter Phys., 2009, 79, 061305.

62 A. Donev, I. Cisse, D. Sachs, E. Variano, F. Stillinger, R. Connelly, S. Torquato and P. Chaikin, Science, 2004, 303, 990.

63 G. Delaney, D. Weaire, S. Hutzler and S. Murphy, Philos. Mag. Lett., $2005, \mathbf{8 5}, 89$.

64 G. W. Delaney, S. Hutzler and T. Aste, Phys. Rev. Lett., 2008, 101, 120602.

65 G. W. Delaney and P. W. Cleary, Europhys. Lett., 2010, 89, 34002.

66 A. Wouterse, S. Luding and A. Philipse, Granular Matter, 2009, 11, 169.

67 The entries in this map are proportional to the X-ray attenuation coefficient of the material which is in turn related to the material density.

68 Such polydispersity was estimated by the distribution of the weight of a sub set of 200 grains for each bead kind and by direct observation of a small set of grains by microscopy. The results are consistent with the producer's specifications. An indirect measure of polydispersity is given in Fig. 4 (see caption), where the resulting data are indeed consistent with the measured values.

69 Eqn (5) assumes spherical symmetry for $g(r)$. We verified that this is indeed the case in these samples where we do not observe any significant orientation dependence in the statistics of the grain distribution..$^{9,25,28}$ 